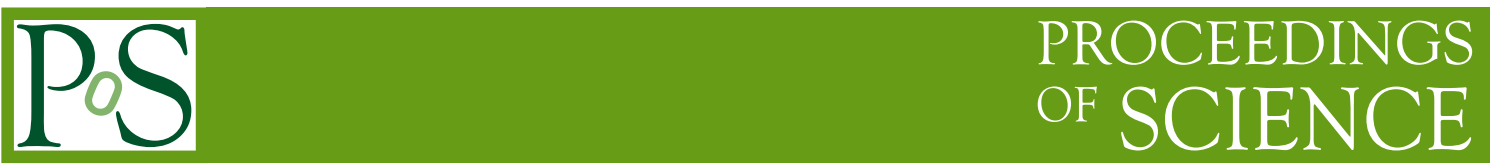

\title{
Multijet measurements with the CMS detector at 7 TeV
}

\section{Panagiotis KOKKAS*广}

University of Ioannina

E-mail: pkokkas@uoi.gr

\begin{abstract}
Measurements sensitive to QCD multijet production are presented, using data from proton-proton collisions at a centre-of-mass energy of $7 \mathrm{TeV}$ collected with the CMS detector at the CERN LHC. Data were collected during the 2010 data-taking period, and correspond to an integrated luminocity of up to $36 \mathrm{pb}^{-1}$. The dijet azimuthal decorrelations, the hadronic event shapes and the ratio of the 3-jet to 2-jet production cross-sections confront the QCD multijet dynamics at a previously unexplored kinematic regime. The data are compared to the predictions of various QCD Monte-Carlo generators.
\end{abstract}

The 2011 Europhysics Conference on High Energy Physics-HEP 2011,

July 21-27, 2011

Grenoble, Rhône-Alpes France

\footnotetext{
* Speaker.

${ }^{\dagger}$ For the CMS Collaboration.
} 


\section{Introduction}

In leading-order (LO) perturbative Quantum Chromodynamics ( $\mathrm{pQCD}$ ), jet production in pp collisions occurs when two partons interact via the strong force to produce two final-state outgoing partons which manifest themselves as hadronic jets. Events with three or more jets in the final state originate from hard-gluon radiation and other higher-order QCD processes. The understanding of multijet production is of great inportance because QCD-induced processes constitute, in some cases, the dominant backgrounds for signals of new physics at the LHC.

In this paper, three new measurements sensitive to QCD multijet production are reported, using data collected with the CMS detector [1] during 2010. The dijet azimuthal decorrelations, the hadronic event shapes and the ratio of the 3-jet to 2-jet production cross-sections confront the QCD multijet dynamics at a previously unexplored kinematic regime. The data are compared to various QCD Monte-Carlo (MC) generators.

\section{The measurements}

The dijet azimuthal decorrelations can be used to study QCD radiation effects over a wide range of jet multiplicities. The observable chosen, is the differential dijet cross section in the azimuthal separation $\Delta \varphi_{\text {dijet }}$ between the two leading jets, normalized by the dijet cross section integrated over the entire $\Delta \varphi_{\text {dijet }}$ phase space, $\left(1 / \sigma_{\text {dijet }}\right)\left(d \sigma_{\text {dijet }} / d \Delta \varphi_{\text {dijet }}\right)$. The normalized dijet cross section is measured [2] in bins of the leading $p_{T}^{\max }$ using the first $2.9 \mathrm{pb}^{-1}$ of data. The measurement provides an indirect handle on the multijet production since $\Delta \varphi_{\text {dijet }}$ values close to $\pi$ denote back-to-back dijet production, while values $<2 \pi / 3$ denote the multijet regime.

Figure 1, on the left, shows the normalized $\Delta \varphi_{\text {dijet }}$ distributions in several $p_{T}{ }^{\max }$ regions, together with predictions from PYTHIA6, PYTHIA8, HERWIG++, and MADGRAPH MC generators. The predictions from PYTHIA6 and HERWIG++ describe the shape of the data distributions well, while MADGRAPH (PYTHIA8) predicts less (more) azimuthal decorrelation than is observed in the data. The same figure, on the right, shows the ratios of measured normalized $\Delta \varphi_{\text {dijet }}$ distributions to NLO pQCD predictions. The NLO predictions provide a good description of the shape of the data distributions over much of the $\Delta \varphi_{\text {dijet }}$ range. Compared to the data, the reduced decorrelation in the theoretical prediction and the increased sensitivity to the $\mu_{r}$ and $\mu_{f}$ scale variations for $\Delta \varphi_{\text {dijet }}<2 \pi / 3$ are attributed to the fact that the PQCD prediction in this region is effectively available only at leading order, since the contribution from tree-level four-parton final states dominates.

The hadronic event-shape variables provide information about the QCD multijet production. The central transverse thrust is defined as $\tau_{\perp, \mathscr{C}} \equiv 1-\max _{\hat{n}_{\mathrm{T}}} \frac{\sum_{i}\left|\vec{p}_{\perp, i} \hat{n}_{\mathrm{T}}\right|}{\sum_{i} p_{\perp, i}}$ where $p_{\perp, i}$ is the transverse momentum of selected jet $i$. The axis $\hat{n}_{\mathrm{T}}$ which maximizes the sum, and thus minimizes $\tau_{\perp, \mathscr{C}}$, is called the thrust axis $\hat{n}_{\mathrm{T}, \mathscr{C}}$. Two-jet events that are well balanced have low values of this variable, while isotropic multijet events have high values. The measurement [3] has been performed using $3.2 \mathrm{pb}^{-1}$ of data, and for three different momentum bins of the leading jet $p_{T}$.

Figure 2 , on the left, shows the distribution of the logarithm of the central transverse thrust for events with leading jet $p_{T}$ between 125 and $200 \mathrm{GeV} / \mathrm{c}$, from data and Pythia6, Pythia8, MAdGraph, AlPGEN, and Herwig++ MC generators. In the same figure, on the right, the ratio between data and the different MC generators samples is shown. The Pythia6 and HeRwig++ 

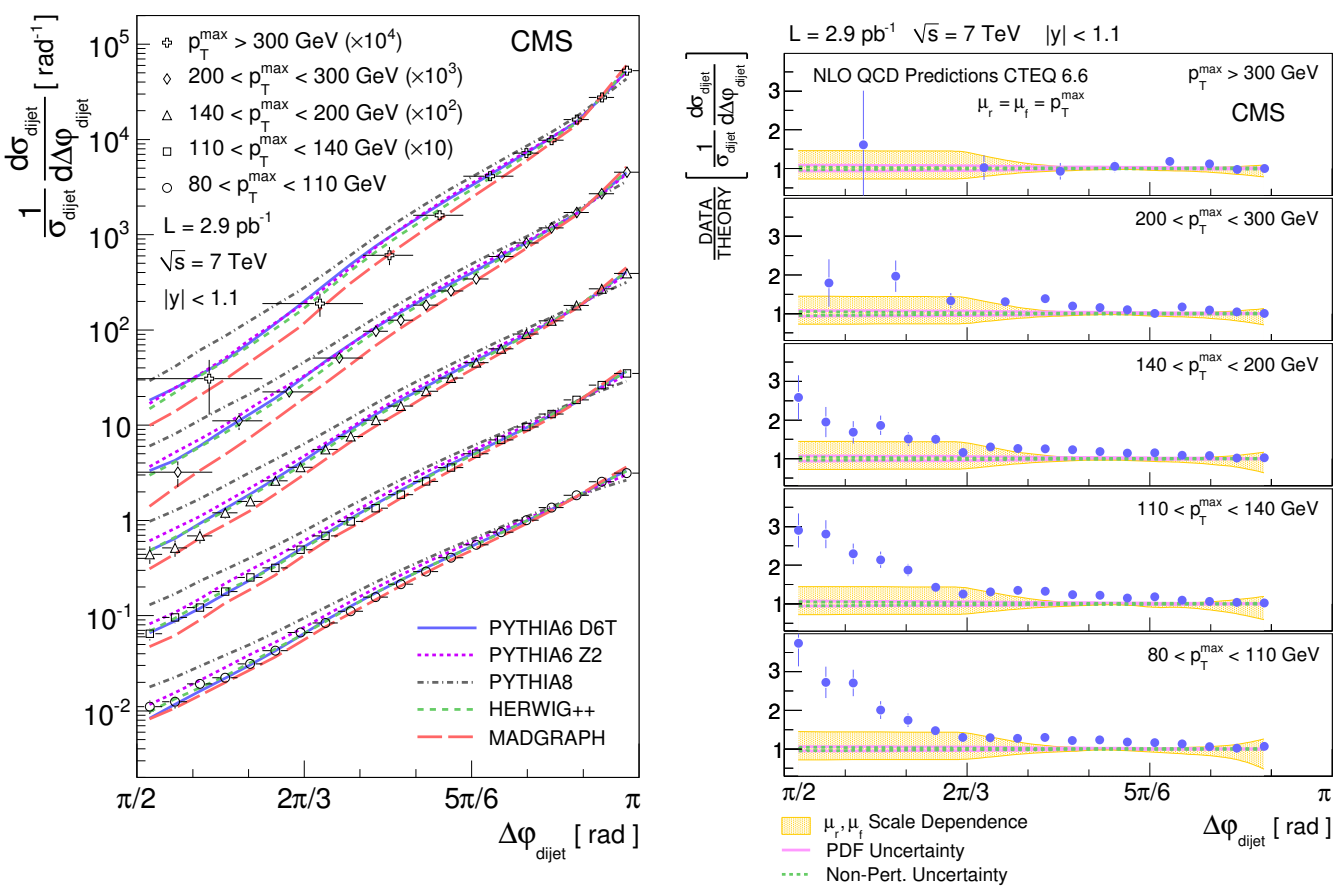

Figure 1: On the left, the normalized $\Delta \varphi_{\text {dijet }}$ distributions in several $p_{T}{ }^{\text {max }}$ regions. The curves represent predictions from four MC generators. The error bars on the data points include statistical and systematic uncertainties. On the right, the ratios of measured normalized $\Delta \varphi_{\text {dijet }}$ distributions to NLO pQCD predictions with non-perturbative corrections in several $p_{T}^{\max }$ regions.
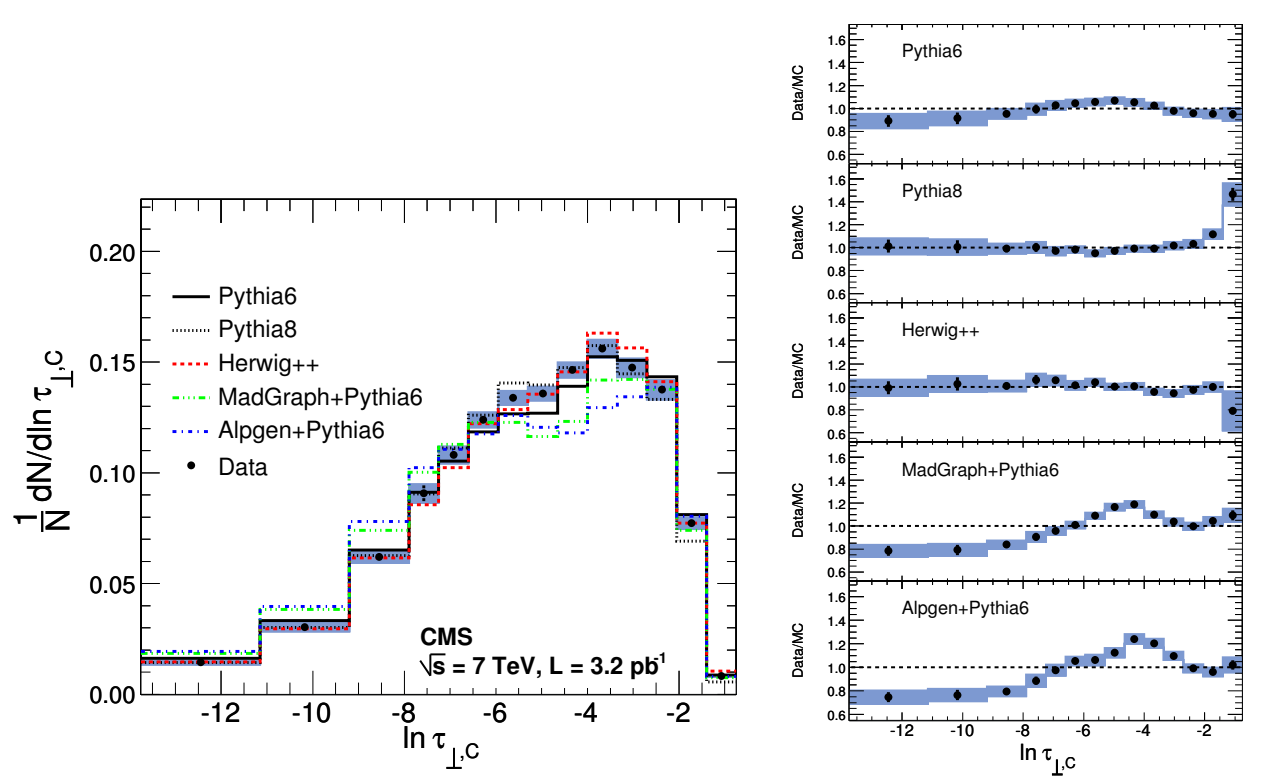

Figure 2: On the left, the distribution of the logarithm of the central transverse thrust for events with leading jet $p_{T}$ between 125 and $200 \mathrm{GeV} / \mathrm{c}$, from data and five MC simulations. The error bars on the data points represent the statistical uncertainty on the data, and the shaded bands represent the sum of statistical and systematic errors. On the right the plots show the ratio between data and the different simulated samples. 
predictions agree with the measurements in all three momentum bins, while the ALPGEN and MADGRAPH curves deviate from the data as a result of an overestimate of the fraction of back-toback dijet events, which enter the lower tail of the distributions. The PYTHIA8 predictions agree with the measurements in all bins of the event-shape variables except in the highest bin, where an underestimate is observed (only $0.5 \%$ of all events).

The measurement $R_{32}$ of the inclusive 3-jet to 2-jet cross section ratio is another direct probe of the multijet production. The measurement has been performed [ [7] using an integrated luminosity of $36 \mathrm{pb}^{-1}$ and expressed as a function of the scalar sum of the jet transverse momenta $H_{\mathrm{T}}=\sum_{i=1}^{N} p_{\mathrm{T}_{i}}$. Figure 3 , on the left, shows the measured $R_{32}$ (solid circles) as a function of $H_{T}$ and the prediction of Pythia6, Pythia8, MadGraph, Alpgen, and Herwig++ (curves) MC generators. In the same figure, on the right, the ratios of the predicted $R_{32}$ value from the MC generators to the measured value is shown. All models considered in this study are consistent with the data for $H_{\mathrm{T}}>0.5 \mathrm{TeV}$. The predictions of MADGRAPH are in agreement with the measured $R_{32}$ throughout the range of this measurement.
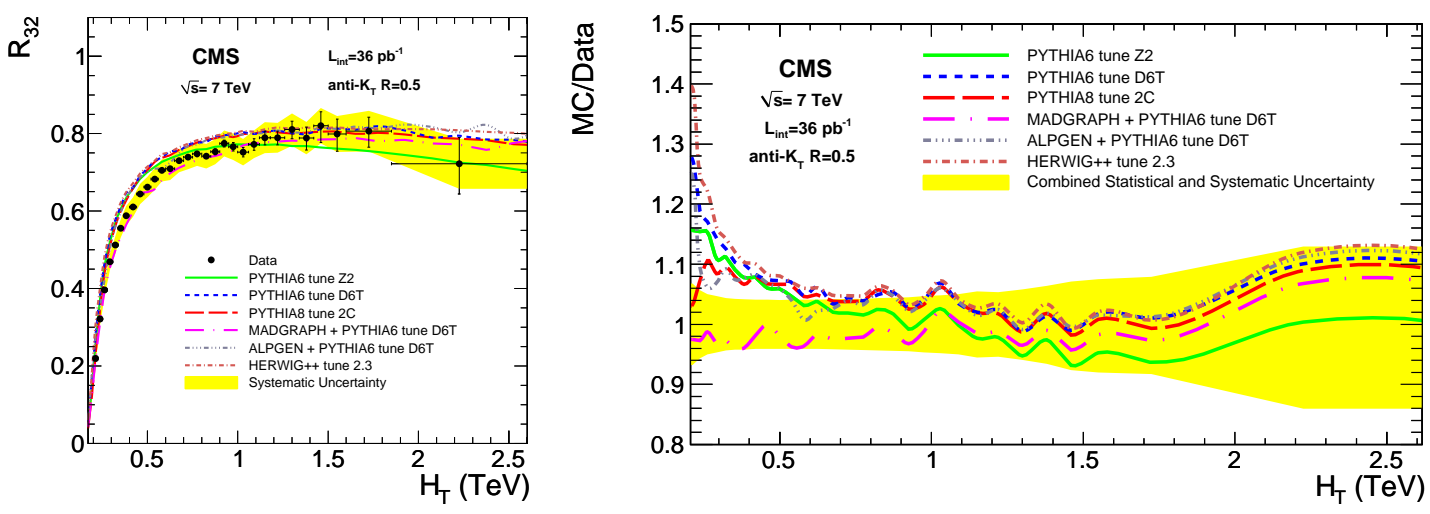

Figure 3: On the left, the measured $R_{32}$ (solid circles) as a function of $H_{T}$ and the prediction of five different MC generators (curves). On the right the ratios of the predicted $R_{32}$ values from the MC generators to the measured value.

\section{Summary}

CMS has performed various QCD multijet studies using the 2010 data from proton-proton collisions at a centre-of-mass energy of $7 \mathrm{TeV}$. The dijet azimuthal decorrelations, the hadronic event shapes and the ratio of the 3-jet to 2-jet production cross-sections confront the QCD multijet dynamics at a previously unexplored kinematic regime. The predictions of various QCD MonteCarlo generators are in good agreement with data. The small discrepancies with data, which have been observed, can be used for further tuning of these widely used MC generators.

\section{References}

[1] CMS Collaboration, JINST 3 (2008) S08004.

[2] CMS Collaboration, Phys. Rev. Lett. 106 (2011) 1222003.

[3] CMS Collaboration, Phys. Lett. B 699 (2011) 48.

[4] CMS Collaboration, Phys. Lett. B 702 (2011) 336. 